\title{
ENSINO DO PROCESSO DE ENFERMAGEM NAS ESCOLAS DE GRADUAÇÃO EM ENFERMAGEM DO ESTADO DE SÃO PAULO ${ }^{1}$
}

Magda Cristina Queiroz Dell'Acqua ${ }^{2}$

Ana Maria Kazue Miyadahira ${ }^{3}$

Dell'Acqua MCQ, Miyadahira AMK. Ensino do processo de enfermagem nas escolas de graduação em enfermagem do estado de São Paulo. Rev Latino-am Enfermagem 2002 março-abril; 10(2):185-91.

Trata-se de um estudo cujo objetivo foi descrever como é oferecido o ensino do processo de enfermagem nas Escolas de Graduação em Enfermagem, do Estado de São Paulo. A população foi composta de 247 docentes. Na metodologia de estudo foi elaborado um questionário, que foi enviado aos 899 docentes das 32 instituições públicas e privadas de Graduação em Enfermagem, do Estado de São Paulo. Dos resultados, em relação ao ensino do processo de enfermagem, do total de 147 docentes que ministravam algum conteúdo, 66 (44,9\%) docentes são de 19 escolas privadas e 81 (55,1\%) docentes, de 8 escolas públicas.

DESCRITORES: processos de enfermagem, ensino superior, enfermagem

\section{TEACHING NURSING PROCESS AT UNDERGRADUATE NURSING PROGRAMS IN THE STATE OF SÃO PAULO}

The goal of this study was to describe the teaching of the nursing process at undergraduate nursing programs in the state of São Paulo. The sample was formed by 247 faculty members. With respect to the study methodology, authors elaborated a questionnaire that was sent to 899 faculty members from 32 public and private nursing schools in the state of São Paulo. Results showed regarding the teaching of the nursing process that, from the total of 147 faculty members who taught the content, 66 (44.9\%) were from 19 private schools and 81 (55.1\%) from 8 public schools.

DESCRIPTORS: nursing process, higher education, nursing

\section{LA ENSEÑANZA DEL PROCESO DE ENFERMERÍA EN LAS ESCUELAS DE PREGRADO EN ENFERMERÍA DEL ESTADO DE SÃO PAULO}

El objetivo del estudio fue describir como es ofrecida la enseñanza del proceso de enfermería en las Escuelas de Graduación en Enfermería del Estado de São Paulo. La población fue de 247 docentes. En la metodología del estudio fue elaborado un cuestionario, que se envió a los 899 docentes de las 32 instituciones públicas y privadas de pregrado de Enfermería del Estado de São Paulo. En los resultados, con relación a la enseñanza del proceso de enfermería, de la totalidad de 147 docentes que ofrecieron algún contenido, 66 (44,9\%) docentes son de 19 escuelas privadas y 81 (55,1\%)son docentes de 8 escuelas públicas.

DESCRIPTORES: procesos de enfermería, enseñanza superior enfermería

\footnotetext{
${ }^{1}$ Trata-se de parte da dissertação apresentada à Escola de Enfermagem da Universidade de São Paulo, 1999. Este estudo foi subvencionado pelo CNPq; ${ }^{2}$ Professor Assistente do Departamento de Enfermagem da Faculdade de Medicina de Botucatu da Universidade do Estado de São Paulo, e-mail: mqueiroz@fmb.unesp.br; ${ }^{3}$ Professor Titular da Escola de Enfermagem da Universidade de São Paulo
} 
INTRODUÇÃO

$D_{\text {urante a vivência profissional, percebeu-se que o método }}$ científico não era utilizado, comumente, pelos enfermeiros, com 0 objetivo de planejar a assistência de maneira clara e intencional ou para determinar e avaliar as ações de enfermagem. Acredita-se que o cuidado seja mais adequado quando o enfermeiro consiga perceber, por meio de sua experiência, de sua habilidade técnica e cognitiva, as reais demandas de cuidado e, assim, elabore propostas sistematizadas, individualizadas e prioritariamente articuladas com as necessidades reais do paciente/cliente e família.

O Processo de Enfermagem é um método para a organização e prestação do cuidado de enfermagem ${ }^{(1)}$. Encontramse, na literatura, outras denominações como Sistematização da Assistência de Enfermagem (SAE) e Metodologia da Assistência de Enfermagem (MAE), termos empregados genericamente, para se referirem à forma de organizar a assistência. No entanto, conceitualmente, não se caracterizam como sinônimos.

O ensino de enfermagem no Brasil teve como referencial a enfermagem americana, que, no início do século, apresentava um ensino com pouco conteúdo teórico e enfocava a repetição de tarefas, sem a esperada compreensão dos fenômenos. Esse quadro fez com que fosse determinado o "Standart Curriculum" (currículo padrão), baseado numa instrução teórica. O "Standart Curriculum for Nursing School", proposto pela primeira vez em 1917, foi revisto em 1927 e em 1937, sendo que, nessa última revisão, foi ressaltado o ensino clínico como essencial ${ }^{(2)}$.

No Brasil, em 1962, houve um marco para o ensino, quando foi determinado o Currículo Mínimo, pelo Conselho Federal de Educação (CFE) - Parecer n $n^{0} 271 / 62^{*}$. Com isso, esperava-se corrigir a fragmentação e as lacunas dos conteúdos, incluindo a fundamentação clínica e a saúde pública. Entretanto, o Parecer $n^{\circ}$ $163 / 72^{* *}$ foi modificado com a Reforma Universitária, Portaria Ministerial $n^{0} 159$, de 14 de julho de 1965, o qual revisou, dentre outros pontos, a duração dos cursos superiores ${ }^{(3)}$.

Sendo assim, pode-se notar que o ensino de enfermagem acompanhou um processo histórico até a publicação da Lei de Diretrizes e Bases da Educação (LDB - 1996) a qual, no artigo 53, inciso II, adota Diretrizes Curriculares, ao invés de currículo mínimo. Com isso, foi possivel flexibilizar a organização e a operacionalização do currículo pleno de graduação, permitindo às Instituições de Ensino Superior que componham seu próprio projeto pedagógico, de acordo com a sua realidade ${ }^{(4)}$.
Nesse momento de transformações e aberturas, o Processo de Enfermagem poderá ser inserido, de forma mais efetiva, nos currículos dos Cursos de Graduação, visto tratar-se de um método que, se utilizado de maneira crítica e reflexiva, poderá contribuir com a resolução de problemas na prática profissional.

0 Processo de Enfermagem tem, na sua estrutura, três grandes dimensões, que são analisadas como: propósito, organização e propriedades. 0 propósito principal do processo é oferecer uma estrutura que atenda às necessidades individualizadas do cliente, família e comunidade, e deve existir uma relação interativa entre 0 foco principal e o enfermeiro. A organização é a dimensão que conta com as cinco fases do processo. Como propriedades, são abordadas seis: ser intencional, sistemático, dinâmico, interativo, flexível e basear-se em teorias ${ }^{(5)}$.

Para a utilização de forma eficiente do processo, há necessidade da aplicação dos conceitos e teorias de enfermagem, das ciências biológicas, físicas, comportamentais e das humanas, para que se tenha um substrato racional ao se tomarem decisões, ao se fazerem julgamentos, nas relações interpessoais e ações de enfermagem ${ }^{(6)}$.

A primeira descrição de processo foi realizada por Lydia Hall, em 1955. Dorothy Johnson (1959), Ida Orlando (1961) e Ernestine Wiedenbach (1963) desenvolveram, cada uma, um modelo diferente, constituído por três fases ${ }^{(1)}$.

Em 1967, Yura e Walsh escreveram o primeiro texto que descrevia quatro fases: histórico, planejamento, implementação e avaliação. Na metade dos anos 70, Bloch (1974), Roy (1975), Mundinger e Jauron (1975) e Aspinall (1976) acrescentaram às 4 fases o diagnóstico, constituindo-se o processo de cinco fases ${ }^{(5)}$.

0 processo de enfermagem foi delineado de maneiras diferentes, pelos diversos autores, mas existem elementos comuns que são: Histórico de Enfermagem ou Avaliação Inicial, Diagnóstico de Enfermagem, Planejamento, Implementação e Evolução de Enfermagem ${ }^{(6-7)}$.

A operacionalização do processo ocorre por meio de seis fases, o histórico de enfermagem; o diagnóstico de enfermagem; 0 plano assistencial; o plano de cuidados ou prescrição de enfermagem; a evolução de enfermagem; o prognóstico ${ }^{(8)}$.

Em nosso meio, após a promulgação da Lei 7.498, de 25/ 06/86, referente ao exercício da enfermagem, que dispõe, no Art. 11, como atividades exclusivas do enfermeiro, a "consulta de enfermagem, a prescrição da assistência de enfermagem, os cuidados diretos de enfermagem a pacientes graves, com risco de vida, como

\footnotetext{
* Parecer $n^{\circ}$ 271/62, aprovado em 19 de outubro de 1962: o Conselho Federal de Educação fixava o currículo mínimo para os Cursos de Enfermagem. Publicado em Documenta $n^{\circ}$ 10, de dezembro de 1962

** Parecer n 163/72, C.C.R. de Currículos, aprovado em 28 de janeiro de 1972. Currículo Mínimo dos Cursos de Enfermagem e Obstetrícia. Publicado em Documenta $n^{0} 135$, de fevereiro de 1972
} 
os cuidados de enfermagem de maior complexidade técnica e que exijam conhecimentos de base científica e capacidade de tomar decisões imediatas" ${ }^{\prime \prime}$.

Sob esse contexto, parte-se da premissa de que a sistematização da assistência de enfermagem deve ser incorporada à prática, como uma possibilidade relevante para cumprir o que orienta e determina a lei do exercício profissional.

No Brasil, o ensino do Processo de Enfermagem, nas escolas de graduação e também em cursos de pós-graduação, teve desenvolvimento acentuado na década de 70 . Ficou registrada a influência de Wanda Horta nesse período, em vários acontecimentos, dentre os quais se ressalta a participação da professora Horta, em 1972, na Escola Ana Néri, que instituía o $1^{\circ}$ Curso de Mestrado em Enfermagem, no Brasil ${ }^{(10)}$.

$\mathrm{Na}$ década de 80 , as pesquisadoras realizaram um estudo sobre o ensino da Metodologia da Assistência de Enfermagem, em seis escolas de graduação da grande São Paulo, com 70 docentes de disciplinas do tronco profissionalizante, e concluíram que este não estava sendo implementado de forma a garantir a competência do futuro profissional para direcionar seu trabalho, tendo por base 0 método científico ${ }^{(11)}$.

De acordo com o exposto, acredita-se na importância da utilização de uma metodologia científica para realizar a assistência de enfermagem individualizada, estreitando as relações profissionais e aumentando o compromisso com o cliente, a família e a comunidade. Existe a necessidade de se elaborarem estudos que tratem do ensino do Processo de Enfermagem, abordando os profissionais docentes e os alunos de graduação em enfermagem.

\section{OBJETIVOS}

Este estudo tem os seguintes objetivos:

1 - Caracterizar o perfil dos docentes que ministram aulas, no tronco profissional nas Escolas de Graduação em Enfermagem, do Estado de São Paulo.

2 - Descrever como é oferecido o ensino do Processo de Enfermagem nas Escolas de Graduação em Enfermagem, do Estado de São Paulo, no que se refere aos conteúdos teóricos e práticos, aos campos de ensino prático, com metodologia implantada, ao referencial teórico, disciplinas, às referências bibliográficas básicas, assim como às fases e formas de avaliação.

\section{MÉTODOS}

População/amostra

A população-alvo ou amostra deste estudo foi constituída pelos docentes das Escolas de Graduação em Enfermagem, do Estado de São Paulo. Segundo os dados fornecidos pelo Comitê de Ensino Superior de Enfermagem, SP (CESE)-apoiado pela ABEn$\mathrm{SP}$, e o Grupo de Investigação em Recursos Humanos Enfermagem (GIRHE)- ligado à Escola de Enfermagem de Ribeirão Preto, no momento havia 32 instituições de ensino com Cursos de Graduação em Enfermagem, sendo designados para esta pesquisa em números de 1 a 32.

Foram enviados questionários a todos os docentes do tronco profissionalizante, das 32 Instituições de Ensino de Graduação em Enfermagem, do Estado de São Paulo, perfazendo um total de 899 sujeitos, 556 docentes de 24 escolas privadas e 343 docentes de 8 escolas públicas. Obteve-se um retorno geral de, aproximadamente, $27,5 \%$ dos questionários enviados, o que resultou em um total de 247 respostas de docentes, de 27 instituições $(83,4 \%)$.

Os critérios para inclusão dos docentes foram: consentir em participar da pesquisa, após os devidos esclarecimentos sobre 0 estudo e ministrarem aulas em disciplinas do tronco profissional, nos Cursos de Graduação em Enfermagem, do Estado de São Paulo.

Coleta de dados

Instrumento

Para a obtenção dos dados, elaborou-se um questionário que foi acompanhado de um ofício construído à luz da resolução 196/96, do Ministério da Saúde, que traz as Diretrizes e Normas Regulamentadoras de Pesquisa, envolvendo seres humanos, esclarecendo sobre os objetivos e os procedimentos que seriam utilizados na pesquisa, para que os sujeitos pudessem sentir-se livres para participar ou não da coleta de dados. 0 projeto foi submetido ao Comitê de Ética em Pesquisa, da FMB-UNESP, e obteve-se, em ofício 077/98 - CEP, parecer favorável.

\section{Operacionalização}

Inicialmente, foi realizado um contato pessoal, por telefone, da pesquisadora com cada diretor, coordenador de curso, chefe de departamento ou secretária acadêmica, para solicitar o endereço completo das instituições, bem como o número de docentes do tronco profissionalizante de cada Escola de Graduação em Enfermagem, do Estado de São Paulo. Nessa oportunidade, foi esclarecida a finalidade da solicitação e os trâmites da pesquisa no Comitê de Ética.

Após o contato pessoal com os representantes das 32 instituições, explicando a finalidade e os objetivos da pesquisa, foi solicitada autorização e colaboração para que os questionários 
enviados fossem distribuídos aos docentes. Encaminhou-se para todas as escolas um pacote via sedex, nominal à pessoa contatada por telefone, contendo os questionários, com os ofícios, em um envelope previamente selado e preenchido com o endereço da pesquisadora, para o retorno.

Coletaram-se dados nos meses de março a julho de 1998, com o auxílio dos representantes de cada instituição.

Tratamento estatístico

Os dados foram eletronicamente compilados, em uma planilha, pela própria pesquisadora e por meio do programa Excel/ 97. Para a análise estatística dos dados, utilizou-se o Software Release 6.12, Statistical Analysis System (SAS).

Fez-se um estudo descritivo, observando-se as freqüências absolutas e percentuais dos dados. Calcularam-se médias e desviospadrão referentes aos percentuais de questionários recebidos.

\section{RESULTADOS E DISCUSSÃO}

\section{Caracterização da população/amostra estudada}

O perfil dos docentes foi determinado pelos aspectos referentes à idade, sexo, tempo de formado, atualização após a graduação, tempo de experiência, regime de trabalho e disciplina na qual atuam. Com o intuito de permitir a compreensão do contexto onde esses docentes estão inseridos, julgou-se oportuno apresentar alguns dados sobre as Instituições de origem, incluindo sua classificação, número de vagas anuais e número de docentes. Das 32 instituições de ensino superior para enfermagem no Estado de São Paulo, a maioria é classificada como escola privada, $24(75 \%)$ e 8 (25\%) como pública. Essa classificação foi realizada conforme orienta o Decreto ${ }^{0} 2.306$, de 19 de agosto de 1997, que classifica as instituições de ensino superior quanto à natureza jurídica em públicas, quando criadas ou incorporadas, mantidas e administradas pela União, e privadas, quando mantidas e administradas por pessoas físicas ou jurídicas de direito privado ${ }^{(12)}$.

O número de docentes por instituições e os questionários que retornaram, bem como o desvio padrão, estão apresentados na Tabela 1.

Tabela 1 - Distribuição de freqüências absolutas e relativas dos questionários respondidos, médias e desvios-padrão, segundo instituição. São Paulo, 1998

\begin{tabular}{|c|c|c|c|c|c|c|c|}
\hline \multicolumn{2}{|c|}{ Institidicônes de origem } & \multicolumn{2}{|c|}{ Docentes por in stithição } & \multicolumn{2}{|c|}{ Questionários } & \multirow{2}{*}{$\frac{\text { Respondidos }}{\%}$} & \multirow[t]{2}{*}{ Média $\pm D P$} \\
\hline & $n^{0}$ & Subtotal & Total & $n^{0}$ & Total & & \\
\hline \multirow{16}{*}{ Públicas } & $\begin{array}{l}1 \\
2\end{array}$ & $\begin{array}{l}79 \\
30\end{array}$ & & $\begin{array}{l}30 \\
24\end{array}$ & & $\begin{array}{l}38,0 \\
80,0\end{array}$ & \\
\hline & 3 & 26 & & 23 & & 80,5 & \\
\hline & 4 & 25 & 343 & 21 & 152 & 84,0 & $520 \% \pm 7,5 \%$ \\
\hline & $\begin{array}{l}5 \\
6\end{array}$ & $\begin{array}{l}63 \\
68\end{array}$ & & $\begin{array}{l}18 \\
17\end{array}$ & & $\begin{array}{l}28,6 \\
250\end{array}$ & \\
\hline & 7 & 27 & & 14 & & 51,9 & \\
\hline & 8 & 25 & & 5 & & 20,0 & \\
\hline & 9 & $4 \overline{2}$ & & 10 & & 23,8 & \\
\hline & 10 & 19 & & 10 & & 52,6 & \\
\hline & 11 & 30 & & 9 & & 30,0 & \\
\hline & 12 & 14 & & g & & 64,3 & \\
\hline & 13 & 30 & & 8 & & 26,7 & \\
\hline & 14 & $\begin{array}{l}17 \\
11\end{array}$ & & 6 & & $\begin{array}{l}35,3 \\
545\end{array}$ & \\
\hline & $\begin{array}{l}15 \\
16\end{array}$ & $\begin{array}{l}11 \\
18\end{array}$ & & $\begin{array}{l}6 \\
5\end{array}$ & & $\begin{array}{l}54,5 \\
27,8\end{array}$ & \\
\hline & 17 & 8 & & 5 & & 62,5 & \\
\hline & 18 & 32 & & 4 & & 12,5 & \\
\hline & 19 & 23 & & 4 & & 17,4 & $22,2 \% \pm 20,5 \%$ \\
\hline \multirow{13}{*}{ Privadas } & 20 & 15 & 556 & 4 & 95 & 26,7 & \\
\hline & 21 & 29 & & 3 & & $\begin{array}{l}10,3 \\
214\end{array}$ & \\
\hline & 23 & $\begin{array}{l}14 \\
8\end{array}$ & & 3 & & $\begin{array}{l}21,4 \\
37,5\end{array}$ & \\
\hline & 24 & 40 & & 2 & & 5,0 & \\
\hline & 25 & 12 & & 2 & & 16,7 & \\
\hline & 26 & 30 & & 1 & & 3,3 & \\
\hline & 27 & 25 & & 1 & & 4,0 & \\
\hline & 28 & 32 & & 0 & & 0,0 & \\
\hline & 29 & 31 & & 0 & & 0,0 & \\
\hline & 30 & 19 & & 0 & & 0,0 & \\
\hline & 31 & 30 & & 0 & & 0,0 & \\
\hline & 32 & & & & & 0,0 & \\
\hline & Total & 899 & & 247 & & $\%$ & \\
\hline
\end{tabular}


Na Tabela 1, observa-se que houve uma média de retorno de $52,0 \%$ e um desvio-padrão de 7,5\% para as escolas públicas; para as escolas privadas, houve uma média de retornos de $22,2 \%$ e um desvio-padrão de $20,5 \%$. Houve, portanto, maior variabilidade no envio de respostas de questionários das instituições privadas. Essa maior variabilidade deveu-se, também, às cinco escolas privadas que, apesar dos esforços realizados por meio de contatos telefônicos, solicitando aos docentes que respondessem, não devolveram os questionários. 0 total geral de respostas foi $n=228$ questionários de docentes, já excluídos os 19 questionários de escolas públicas que retornaram totalmente em branco.

Quanto ao número de vagas oferecidas pelas 32 instituições, a faixa que predominou foi de $13(40,6 \%)$ instituições com 70 a 90 vagas, seguida por $7(21,8 \%)$ escolas com 20 a 50 vagas. 0 menor número de vagas foi 20 , e o maior, 700 vagas, oferecidas por uma escola particular que informou realizar dois vestibulares no ano; segundo o número de vagas, mostrou-se que as escolas privadas têm um número reduzido de docentes por instituição, quando comparado com o das escolas públicas, e também concentram os maiores números de vagas anuais.

A faixa etária predominante foi de 30 a 40 anos, com 125 $(55,6 \%)$ docentes; seguiu-se a faixa de 40 a 50 anos, com 73 (32,5\%); $7(3,1 \%)$, na faixa etária de 20 a 30 anos; e apenas $3(1,3 \%)$ docentes estavam na faixa de 60 a 70 anos. 0 docente mais novo referiu ter 20 anos, e o mais velho, 67 anos.

Dos 228 docentes, $218(95,6 \%)$ eram do sexo feminino, e apenas $10(4,4 \%)$, do sexo masculino. Esse resultado já era esperado, pois faz parte do contexto profissional.

Em relação ao tempo de formado, do total de 228 docentes, 4 não informaram, e, entre os 224 que informaram, predominou o período de 10 a 20 anos, referido por 147 (65,6\%) docentes.

Os dados sobre faixa etária, predominando de 30 a 40 anos, com 55,6\%; sexo com $95,6 \%$ e tempo de formado, de 10 a 20 anos, $65,6 \%$, permitiram caracterizar o perfil dos docentes.

Quanto à atualização dos docentes após a graduação, realizando cursos "lato sensu", verificou-se que, do total de 228 docentes, $220(96,4 \%)$ referiram tê-los cursado, sendo que 202 $(91,8 \%)$ fizeram especialização, e 18 (8,2\%), aperfeiçoamento, totalizando 283 cursos de especialização e 18 cursos de aperfeiçoamento.

Com relação à formação de docentes em Pós-Graduação "stricto sensu", do total de 228 docentes do estudo, 191 (83,7\%) têm mestrado, e 106 (46,5\%) têm doutorado.

Observou-se pelos dados que 66 (29,0\%) docentes tinham entre 5 e 10 anos de experiência no ensino de graduação em enfermagem, sendo este o maior percentual encontrado. Também se notou que $54(23,7 \%)$ tinham entre 1 e 5 anos de experiência.
Dos 95 docentes das escolas privadas, a maioria, 74 $(77,9 \%)$ docentes, trabalhava em regime de tempo parcial, variando o número de horas do contrato de trabalho e ficando o docente sujeito às determinações da escola, no que se refere às atividades a serem desempenhadas. 0 inverso ocorre para as escolas públicas, com $130(97,8 \%)$ docentes que atuavam em regime de tempo integral.

Para compreender as atividades exigidas no trabalho docente, utilizou-se a definição de regime de trabalho em tempo integral como sendo aquele com obrigações de prestar 40 horas semanais de trabalho, na mesma instituição, reservando, pelo menos vinte horas semanais, destinadas a estudos, pesquisa, trabalhos de extensão, planejamento e avaliação ${ }^{(12)}$.

Dados relacionados ao ensino do processo de enfermagem

Do total de 228 docentes, participantes do estudo, 147 $(64,5 \%)$ ministravam o processo de enfermagem, sendo que destes, $81(55,1 \%)$ são das escolas públicas, e 66 (44,9\%), das escolas privadas.

Quanto ao conteúdo do processo de enfermagem, optouse por descrever e analisar essa variável de forma distinta para os dois tipos de instituições, pública e privada. Com isso, obteve-se que, dos 66 docentes de escolas privadas que ministravam algum conteúdo, foram levantadas respostas de 63 docentes, sendo que a maioria, 47 (74,6\%), ministrava-o sob a forma teórico-prática, 10 $(15,9 \%)$ docentes somente ofereceram o conteúdo prático, e 6 (9,5\%) docentes ofereceram o conteúdo somente teórico.

Para os 81 docentes das escolas públicas que ofereceram algum conteúdo do processo, 70 responderam qual o conteúdo ministrado e, destes, 51 (72,8\%) ministravam o conteúdo de forma teórico-prática, $17(24,3 \%)$ docentes somente prática, e 2 (2,9\%) docentes somente teórica.

Considera-se que a forma mais adequada para ministrar o conteúdo do processo de enfermagem é a abordagem da teoria e da prática realizadas pelo mesmo docente. Isso permitiria continuidade, coerência e inter-relação do tema, introduzido na teoria e aplicado à prática. Para os docentes que não ministram especificamente 0 conteúdo teórico do processo de enfermagem, é recomendado que 0 utilizem enquanto metodologia para ensinar nas diferentes disciplinas do tronco profissional. A disciplina que apareceu com maior frequêencia, que se ensina no processo de enfermagem, foi "saúde do adulto", com 45 (30,6\%) citações, seguida por $15(10,2 \%)$ em "saúde pública", e com o mesmo percentual, "semiologia e semiotécnica".

Dos docentes que ministram o conteúdo do processo de enfermagem, em campos de estágios, com a metodologia já implantada, para surpresa da autora deste trabalho, a maioria, 49 
(63,6\%) docentes, é de escolas privadas, e 28 (36,4\%), de escolas públicas. Em geral, as instituições públicas têm hospitais governamentais/escolas como campo para seus estágios. Esperavase que se pudesse facilitar a implantação da metodologia da assistência, mas isso não foi encontrado nesta amostra do estudo.

Um aspecto levantado, em trabalho realizado sobre a necessidade e praticabilidade atribuídas à metodologia do processo de enfermagem, proposto por Horta, sendo a população de enfermeiros formada no período de 1975 a 1979, atuando em hospitais, foi a não incorporação dessa metodologia de assistência pelos profissionais de campo, comprometendo a qualidade da assistência prestada, a própria qualidade do trabalho do enfermeiro e acentuando o distanciamento entre Escola e Serviço, reconhecidamente prejudicial para a formação do futuro profissional ${ }^{(13)}$. A mesma autora apresentou a dissociação existente entre a teoria e a prática, sendo que, para esse estudo, concluiu-se que, dos 257 enfermeiros, a maioria, 88,7\%, considerava necessária a utilização da metodologia do processo de enfermagem.

Obtiveram-se resultados bastante diversificados, quanto ao referencial teórico adotado como suporte ao ensino do processo de enfermagem.

Quanto ao referencial teórico, a referência básica mais citada, utilizada enquanto suporte para a sistematização da assistência desenvolvida, foi Horta, com 77 (52,0\%) citações de docentes, o que é coerente, já que esse modelo teórico é o mais conhecido no Brasil, como se evidenciou em trabalho com $73,5 \%$ da amostra $^{(14)}$. Mesmo com diferença de, aproximadamente, 11 anos entre as pesquisas, verificou-se resultado semelhante, no presente estudo.

No que tange às fases do processo de enfermagem, embora grande parte das referências abordem cinco para o método, optouse pelas 6 fases descritas por Horta, por ser a mais conhecida entre os enfermeiros.

Das 6 fases questionadas no estudo, verificou-se 0 seguinte: Histórico de Enfermagem, 83,7\%; Prescrição de Enfermagem, 76,9\%; Evolução de Enfermagem, 76,2\%; Diagnóstico de Enfermagem, 64,0\%; Plano Assistencial, 51,0\% e Prognóstico de Enfermagem, 18,4\%.

Pode-se observar que as três fases mais citadas compõem o denominado "processo simplificado" utilizado em alguns serviços, como forma de sistematizar e registrar o planejamento da assistência de enfermagem. Cabem ressaltar as considerações feitas em estudo sobre o processo de enfermagem, numa unidade de queimados, que propuseram adaptar a Taxonomia I de Diagnósticos de Enfermagem, proposta por NANDA, ao Modelo Conceitual de Horta, e que, por meio dessa experiência, concluíram que modificações podem ser feitas, tornando o modelo viável, sem, contudo, distanciá-lo do referencial $^{(15)}$.
Em relação às estratégias utilizadas em sala de aula e no laboratório de enfermagem, para o ensino do processo de enfermagem, a maioria das citações, $138(53,2 \%)$, foi utilizada por meio de aulas expositivas; em seguida, apareceram 83 (32,0\%) referentes à demonstração e, por último, à dramatização, com 38 $(14,8 \%)$ citações, como recursos para se transmitirem os conteúdos. Verificou-se, então, que houve em $\bar{X}=1,76$ estratégias por docente.

Observa-se que uma das maneiras para facilitar o ensino, além da prática já discutida nesses casos, seria o acesso a materiais didáticos como, por exemplo, vídeos auto-instrucionais e videoconferências.

Quanto às estratégias utilizadas no campo clínico, ou estágio, a mais freqüente foi $129(50,0 \%)$ de supervisão da prática realizada pelo próprio docente, seguida da estratégia, demonstração, na prática, pelo docente, com 36 (14,0\%), e a prática supervisionada pelo enfermeiro de campo, com 28 (10,8\%). As avaliações teóricas totalizaram 167 formas e, desse total, as mais freqüentes, 84 (50,3\%) citações, foram por meio de análise de situações. Quanto às avaliações práticas, eram mais freqüentemente as realizadas através de situações no campo clínico, com 79 (75,2\%) citações dos docentes.

\section{CONCLUSÕES}

O estudo permitiu as seguintes conclusões:

1. Quanto ao perfil dos docentes que ministram aulas no tronco profissional de enfermagem, nas escolas de graduação em enfermagem, do Estado de São Paulo: a maioria, 218 (95,6\%) docentes, é do sexo feminino, na faixa etária de 30 a 40 anos, com $125(55,6 \%)$ docentes, e o tempo de formado, 10 a 20 anos, foi 0 percentual mais elevado, com 147 (65,6\%) docentes. Realizou cursos "lato sensu" a maioria dos docentes, 220 (96,4\%), e, quanto à pósgraduação "stricto sensu", 191 (83,7\%) docentes fizeram mestrado, e 106 (46,5\%), o doutorado. Tempo de experiência em curso de graduação em enfermagem, 66 (29,0\%) docentes tinham de 5 a 10 anos de experiência. O regime de trabalho dos docentes por instituição foi $74(77,9 \%)$ docentes de escolas privadas, em regime de tempo parcial, e $130(97,8 \%)$ docentes de escolas públicas, em regime de tempo integral.

2. Em relação ao ensino do processo de enfermagem, 66 (44,9\%) docentes são de 19 escolas privadas, e 81 (55,1\%) docentes são de 8 escolas públicas. 0 conteúdo teórico-prático foi ministrado por 47 $(74,6 \%)$ docentes de escolas privadas e por $51(72,8 \%)$ docentes de escolas públicas; o conteúdo prático foi ministrado por 10 (15,9\%) docentes de escolas privadas e $17(24,3 \%)$ docentes de escolas públicas, e o conteúdo teórico foi oferecido por $6(9,5 \%)$ docentes de escolas privadas e 2 (2,9\%) docentes de escolas públicas. Os locais 
de estágios com alguma metodologia assistencial implantada foram mais freqüentemente citados por 49 (63,6\%) dos docentes das escolas privadas. A disciplina em que, com maior freqüência, se ministra o conteúdo do processo de enfermagem foi saúde do adulto, com 45 $(30,6 \%)$ citações; 0 referencial teórico mais adotado foi o de HORTA, em 77 (52,0\%) citações. Das 6 fases do processo de enfermagem ensinadas, os maiores percentuais foram $83,7 \%$ para o histórico de enfermagem, $76,9 \%$ para prescrição de enfermagem; $76,2 \%$ para evolução de enfermagem, $64,0 \%$ para diagnóstico de enfermagem, $51,0 \%$ para plano assistencial e $18,4 \%$ para prognóstico. As estratégias em sala de aula e nos laboratórios de enfermagem mais

\section{REFERÊNCIAS BIBLIOGRÁFICAS}

1. Potter PA, Perry AG. Fundamentals of nursing: concepts, process, and practice. 2.ed. St. Louis: Mosby; 1989.

2. Carvalho AC. Orientação e ensino de estudantes de enfermagem no campo clínico. [tese]. São Paulo(SP): Escola de Enfermagem/ USP; 1972.

3. Angerami ELS, Gomes DLS, Mishima SM, Hayashida M, Ribeiro CMM, Reis JN. Estudo comparativo da nomenclatura das matérias e disciplinas do currículo mínimo com a utilizada pelas escolas de enfermagem da região sudeste - Brasil. Rev Latinoam Enfermagem 1996; 4(1):31-46.

4. Martins RO. É hora de estabelecer as Diretrizes Curriculares. Olho mágico 1998;(16):34-5.

5. Iyer PW, Taptich BJ, Bernocchi-Losey D. O processo de enfermagem. In: Iyer PW, Taptich BJ, Bernocchi-Losey D. Processo e diagnóstico em enfermagem. Porto Alegre: Artes Médicas; 1993. p. $1-16$

6. Stanton M, Paul C, Reeves JS. Um resumo do processo de enfermagem. In: George JB. Teorias de enfermagem: os fundamentos para a prática profissional. Porto Alegre: Artes Médicas; 1993. p. 2437.

7. Smeltzer SC, Bare BC. Reflexão crítica e o processo de enfermagem. In: Smeltzer SC, Bare BC. Brunner \& Suddarth: tratado de enfermagem médico-cirúrgica. 8.ed. Rio de Janeiro: Guanabara Koogan; 1998. p. 22-33. citadas foram $138(53,2 \%)$ citações para aulas expositivas, e campo clínico ou estágio foi a prática supervisionada por docentes, com 129 $(50,0 \%)$ citações. A referência bibliográfica básica mais utilizada foi o Processo de Enfermagem de HORTA, com 81 (37,9\%) citações. Quanto às formas de avaliações teóricas e práticas, as mais freqüentes foram a análise de situações e análise de desempenho no campo prático.

Este estudo permitiu descrever o perfil dos docentes que ministram disciplinas da área profissionalizante, bem como identificar as fases e estratégias de ensino do Processo de Enfermagem, nos cursos de graduação em enfermagem do Estado de São Paulo.

8. Horta WA. Processo de enfermagem. São Paulo: EPU.; 1979.

9. Lei n. 7498 de 25 de julho de 1986. Dispõe sobre a regulamentação do exercício da enfermagem e dá outras providências. D.O.U.1986 jul.26:seção 1; 9273-5.

10. Meirelles ASV. O quê e porque do ensino da metodologia assistencial em enfermagem. Rev Esc Enfermagem USP 1987; 21(n.esp.):55-61.

11. Silva SH, Santos LCR, Araujo TL, Laganá MTC. Ensino da metodologia de assistência de enfermagem nas escolas de graduação em enfermagem da grande São Paulo, 1986. Rev Esc Enfermagem USP 1988; 22(n. esp.):189-221.

12. Decreto n. 2.306 de 19 de agosto de 1997. Regulamenta, para 0 Sistema Federal de Ensino as disposições contidas no art. 10 da medida Provisória n. 1.477-39, de 8 de agosto de 1997, e nos arts. 16, 19, 20, 45, 46 e $\S 1^{\circ}, 52$, parágrafo único, 54 e 88 da Lei n. 9394, de 20 de dezembro de 1996. D.O.U. 1986 dez 23: seção 1; 27833. 13. Gutierrez MGR. Necessidades e praticabilidade atribuídas à metodologia do processo de enfermagem proposto por Horta. [dissertação]. São Paulo (SP): Escola de Enfermagem/USP; 1981. 14. Horr L, Gonçalves LHT, Saupe R. O ensino da metodologia assistencial de enfermagem-departamento de enfermagem UFSC. Rev Esc Enfermagem USP 1987; 21:2(n.esp.):1-8.

15. Rossi LA, Dalri MCB. Processo de enfermagem numa unidade de queimado: análise e proposta de reformulação segundo o modelo conceitual de Horta e taxonomia I dos diagnósticos de enfermagem de Nanda. Rev Esc Enfermagem USP 1993;27(3):328-54. 